\title{
Cholinergic control of visual categorization in macaques
}

\author{
Nikolaos C. Aggelopoulos ${ }^{1}{ }^{*}$, Stefanie Liebe ${ }^{1,2}$, Nikos K. Logothetis ${ }^{1}$ and Gregor Rainer ${ }^{1,3}$ \\ Max Planck Institute for Biological Cybernetics, Tuebingen, Germany \\ ${ }^{2}$ Department of Neuroscience, Pharmacology and Physiology, University College London, University of London, London, UK \\ ${ }^{3}$ Visual Cognition Laboratory, Department of Medicine/Physiology, Université de Fribourg, Fribourg, Switzerland
}

Edited by:

Anita Disney, Salk Institute for

Biological Studies, USA

Reviewed by:

Miou Zhou, University of California,

Los Angeles, USA

Janita Turchi, The National Institute of

Mental Health, USA

*Correspondence:

Nikolaos C. Aggelopoulos, Max

Planck Institute for Biological

Cybernetics, Spemannstr. 38, 72076

Tuebingen, Germany.

e-mail: aggelopoulos@tuebingen.

mpg.de
Acetylcholine (ACh) is a neurotransmitter acting via muscarinic and nicotinic receptors that is implicated in several cognitive functions and impairments, such as Alzheimer's disease. It is believed to especially affect the acquisition of new information, which is particularly important when behavior needs to be adapted to new situations and to novel sensory events. Categorization, the process of assigning stimuli to a category, is a cognitive function that also involves information acquisition. The role of ACh on categorization has not been previously studied. We have examined the effects of scopolamine, an antagonist of muscarinic ACh receptors, on visual categorization in macaque monkeys using familiar and novel stimuli. When the peripheral effects of scopolamine on the parasympathetic nervous system were controlled for, categorization performance was disrupted following systemic injections of scopolamine. This impairment was observed only when the stimuli that needed to be categorized had not been seen before. In other words, the monkeys were not impaired by the central action of scopolamine in categorizing a set of familiar stimuli (stimuli which they had categorized successfully in previous sessions). Categorization performance also deteriorated as the stimulus became less salient by an increase in the level of visual noise. However, scopolamine did not cause additional performance disruptions for difficult categorization judgments at lower coherence levels. Scopolamine, therefore, specifically affects the assignment of new exemplars to established cognitive categories, presumably by impairing the processing of novel information. Since we did not find an effect of scopolamine in the categorization of familiar stimuli, scopolamine had no significant central action on other cognitive functions such as perception, attention, memory, or executive control within the context of our categorization task.

Keywords: macaque, learning, categorization, cognition, cognitive, muscarinic, acetylcholine, scopolamine

\section{INTRODUCTION}

The nervous system is adaptive, so that its neuronal properties can be modified by learning to respond to new categories of stimuli. Acetylcholine $(\mathrm{ACh})$ is one of several neuromodulators implicated in several cognitive functions. The nucleus basalis of Meynert (NBM) supplies the non-intrinsic cholinergic input to the neocortex and shows marked cell loss in Alzheimer's patients (Perry, 1988; Levy, 1996) and in other cognitive disorders, for example Wernicke-Korsakoff syndrome (Arendt et al., 1983; Savage et al., 1999) and Creutzfeldt-Jakob disease (Arendt et al., 1984). Indeed ACh is believed to play an important role in many cognitive functions, including attention (Voytko et al., 1994; McGaughy et al., 1996; Sarter and Bruno, 2000; Furey et al., 2008; Herrero et al., 2008; Deco and Thiele, 2010), signal detection (Sillito and Kemp, 1983; Zinke et al., 2006; Goard and Yang Dan, 2009), cue detection (Parikh and Sarter, 2008), decision-making (Roberts et al., 1990), learning (Richardson and DeLong, 1988; Roberts et al., 1990; Sarter et al., 2003), memory encoding (Bakin and Weinberger, 1996; Miasnikov et al., 2001, 2008, 2009; Weinberger, 2003) short-term memory (Miller and Desimone, 1993; Fransen et al., 2006; Plakke et al., 2008; Thomas et al., 2008), and long-term memory retrieval (Rosier et al., 1999; Sarter et al., 2003).

Considering the variety of cognitive functions affected by acetylcholine, the question arises whether some functional specificity can be ascribed to its action. Several studies have tried to dissect the exact system that scopolamine affects, as an effect on one cognitive system can influence others. It has been hypothesized that the cortical cholinergic inputs optimize the processing of signals in attention-demanding contexts (Sarter et al., 2005). Similarly, it has been also hypothesized that cortical cholinergic inputs are involved in the mediation of top-down effects such as the knowledge-based augmentation of "detection of signals," defined according to Posner as the awareness or behavioral report of signals (Posner, 1980; Posner et al., 1980). Actions specifically through muscarinic ACh receptors have been linked to attentional mechanisms (Ruotsalainen et al., 2000). In addition, or in contrast, to the hypothesis of a role in attention, there is considerable evidence for the involvement of muscarinic receptors in visual recognition using delayed non-match to sample (DNMS) tasks. The muscarinic antagonist scopolamine affects the encoding of new information into long-term memory using the Wisconsin General Test Apparatus (Ridley et al., 1984) and reduces the choice accuracy in stimulus-recognition memory forgetting tasks (e.g., DNMS, Aigner and Mishkin, 1986). However, increased forgetting is not dependent on retention interval (Aigner and Mishkin, 1986) which has also been interpreted as an impediment in the entrance of information into memory, i.e., memory encoding or memory storage (Taffe et al., 1999). Another DNMS study by 
Aigner et al. (1991) argued that initial storage but not long-term memories, attentive or perceptual processes are affected by scopolamine, because the monkeys were not affected when injected after acquisition. A study by Myers et al. (2002) suggested up to a $40 \%$ drop in DNMS performance following scopolamine injections while in a different study there was approximately a $12 \%$ performance drop in a DNMS task after scopolamine injection in perirhinal cortex (Tang et al., 1997). A similar deficit with immediate and delayed word recall was seen in human subjects (Ebert et al., 1998). There seems, therefore a relative consensus to have emerged that ACh affects cognition when behaviorally significant or novel stimuli need to be processed (Pepeu and Giovannini, 2004).

Nonetheless, even within this context there has been some debate. For example the study of Voytko et al. (1994) attributed a role in attention to $\mathrm{ACh}$, and more specifically to the NBM cholinergic projection, rather than in learning and memory, based on negative results in a variety of mnemonic tasks. In a separate experiment, the authors interpreted the decrease in correct performance under scopolamine also as an effect of attention, while there was no direct comparison between novel and familiar stimuli, a potential confound when attributing the deficit to attention. A role of ACh in attention has received widespread support among other researchers (e.g., Sarter and Parikh, 2005; Hasselmo and Sarter, 2011) based partly on its role in signal detection (Herrero et al., 2008; Deco and Thiele, 2010).

In contrast to these studies that attempted to define a specific function affected by scopolamine, a more recent study in humans claimed an effect on a variety of cognitive functions including perception, attention, learning, short-term memory, and even recall (Fredrickson et al., 2008). This particular study, however, despite its other advantages, failed to use a control for the peripheral actions of scopolamine, making interpretation of the results difficult, since autonomic depression can impair psychomotor function, attention, and memory recall (Heims et al., 2006).

Categorization involves many cognitive functions that include the perception of a stimulus, motivational state, learning, and its executive allocation to a category. We have examined the effects of scopolamine, an antagonist of muscarinic ACh receptors, on visual object categorization in macaques. An important control in this design was the comparison of categorization performance between novel stimuli and familiar stimuli. If scopolamine had an effect in a variety of cognitive functions other than the categorization specifically of novel stimuli, then the categorization of familiar stimuli should be affected (e.g., due to effects in perception, attention, motivational state, decision, and long-term memory recall, etc.). An additional crucial control was the use of an analog of scopolamine that does not cross the blood brain barrier, in order to dissect the direct cognitive effects through the central action from any indirect effects due to scopolamine's actions on the parasympathetic system.

\section{MATERIALS AND METHODS SUBJECTS AND PHARMACOLOGICAL TREATMENTS}

The experiments described here were carried out in accordance with the EC Directive 86/609/EEC for animal experiments. Two adult male macaques (K03, $12.5 \mathrm{~kg}, 11$ years of age and
D07, $14.5 \mathrm{~kg}, 8$ years of age) were trained in a categorization task: to classify stimuli into categories by appropriate behavioral responses. The paradigm involved a task during which an image that belonged to one of the categories was presented. The macaque used levers to categorize the stimulus. Images were presented at the center of fixation once the macaque pressed a pair of levers for $500 \mathrm{~ms}$. The images remained on the screen for $200 \mathrm{~ms}$ after which period a light gray cue square was presented for $1200 \mathrm{~ms}$ (Figure 1). While the cue square was present, the monkeys could release one of the levers to indicate which category had been shown and, if correct, obtain a juice reward.

On alternate days the macaque received s.c. (subcutaneously) either an injection of scopolamine in $1 \mathrm{ml}$ saline or the vehicle. For example, if a scopolamine injection was made on a Monday, a saline injection would be made on Tuesday. Scopolamine was delivered at 7.5 or $10 \mu \mathrm{g} / \mathrm{kg}$. These doses are similar to those used in human studies (Fredrickson et al., 2008) and in previous studies of rhesus macaques (Taffe et al., 1999; Myers et al., 2002; Plakke et al., 2008). In pilot experiments, lower doses of $5 \mu \mathrm{g} / \mathrm{kg}$ caused drowsiness while higher doses $(15 \mu \mathrm{g} / \mathrm{kg})$ caused some agitation. Testing began $1 / 2 \mathrm{~h}$ to $1 \mathrm{~h}$ after injection.

Scopolamine can cross the blood brain barrier, however it can also cause parasympathetic depression peripherally which could affect either the perception of the stimuli or performance due to some non-specific effects. For example, scopolamine causes pupillary dilation, depression of salivation, suppression of pharyngeal motility, (hence food and juice ingestion) and suppression in the secretion of gastric fluids. In principle, changes in task performance could be centrally mediated and/or peripherally mediated. Butyl-scopolamine, an analog of scopolamine that does not cross the blood brain barrier, was therefore administered as a control, to determine the extent to which the peripheral actions of scopolamine affected performance in the categorization task. Pupillary dilation was used as a bioassay to titrate the effective dose of butylscopolamine at the same level as the doses of scopolamine used in this study. This was achieved with butyl-scopolamine doses of $4.0-6.0 \mathrm{mg} / \mathrm{kg}$.
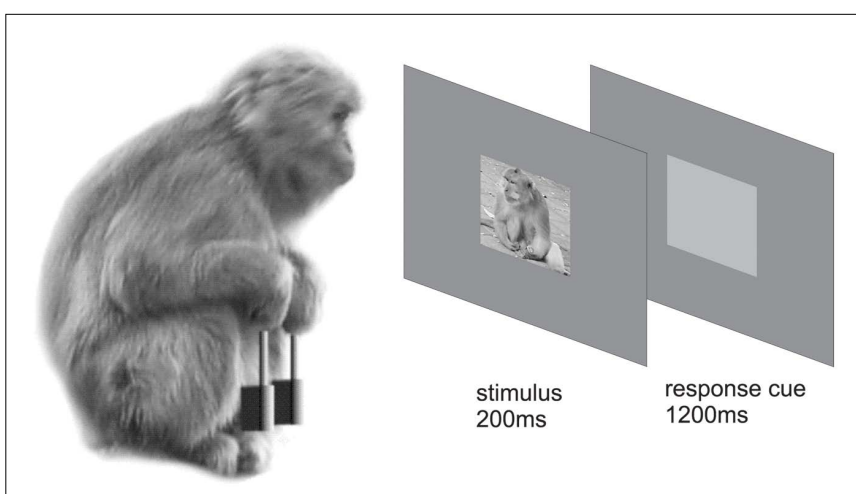

FIGURE 1 | Categorization task. After successfully fixating on a central fixation spot and concurrently pressing two levers for $500 \mathrm{~ms}$, a stimulus was presented for $200 \mathrm{~ms}$. Immediately afterward, a light gray response cue was presented for $1200 \mathrm{~ms}$ during which the macaque had the opportunity to categorize the previously seen image as a "flower" or a "monkey" by releasing the appropriate lever. 
Experiments under butyl-scopolamine started 3 weeks after the end of the scopolamine experiments in the case of D07 and 5 months after the scopolamine experiments in the case of K03. In statistically comparing the difference in performance of scopolamine from butyl-scopolamine in these two separate periods, we have normalized the performance against the saline experiments carried out on the day before or after each drug experiment. Therefore what is compared in Table 2 and the related statistics is the difference in task performance under scopolamine vs. saline from the difference in task performance under butyl-scopolamine vs. saline.

\section{STIMULI AND CATEGORIZATION TASK}

The size of the visual stimulus presented in the categorization task was $6^{\circ}$ of visual angle and the fixation window was $12^{\circ}$. Grayscale stimuli normalized for equal luminance (mean grayscale value $=128$ ), equal contrast, and amplitude spectrum were presented at the center of a computer screen. The luminance of the screen without the stimuli was $7.0 \mathrm{~cd} / \mathrm{m}^{2}$, whereas the mean luminance of the stimuli themselves was $17.8 \mathrm{~cd} / \mathrm{m}^{2}$. The equivalent reflectances were for the screen $3.0 \mathrm{e}^{-2} \mathrm{~W} / \mathrm{sr} . \mathrm{m}^{2}$ and for the stimuli $7.2 \mathrm{e}^{-2} \mathrm{~W} / \mathrm{sr} . \mathrm{m}^{2}$. The stimuli used for the experiment were natural scenes that belonged to either of two categories: monkeys or flowers. The monkey images included in all sessions both macaque and non-macaque images, faces, whole animals, and groups of animals. The flowers were an even mix of radially symmetrical, irregular (e.g., orchids), or several flowers per image at a variety of orientations. The aim was to train the monkeys to use as far as possible true category and not a lower level feature such as a facial feature or radial symmetry.

It has been hypothesized that the cortical cholinergic ACh input is critical in difficult perceptual judgments and optimizes the processing of signals in attention-demanding contexts (Sarter et al., 2005). To improve the probability of seeing an effect of scopolamine on categorization, the images were, therefore, parametrically interpolated with random phase "masks" using a degradation procedure we have used previously (Rainer et al., 2004; Liebe et al., 2009). As individual natural images contain characteristic correlated phase spectra, the procedure increased the noise (i.e., reduced the coherence or phase correlation) of the stimuli (Figure 2). The aim was to introduce graded levels of salience. The levels used were $100,60,55,50,45,40$, and $0 \%$ coherence, with $100 \%$ corresponding to the coherent intact image (after equalization) and 0 to complete noise. We chose this range of coherence because pilot studies had indicated that the macaques had a threshold in their ability to recognize the images in the region of noise levels 40-50. Outside this range, with less noise (over 50\% stimulus coherence) the animals performed as well as with the $100 \%$ intact image whereas with more noise (under $40 \%$ stimulus coherence) their responses were at chance. Every time an image appeared at aiven coherence level it was interpolated with a new random phase mask, so that the image never was quite the same when presented again at the same coherence level. This procedure was introduced so as to avoid a strategy of using diagnostic elements of a stimulus (Nielsen et al., 2006) rather than stimulus category to categorize the images.

The monkeys were trained over several months on a set of six flower and six monkey images (Figure 3), which became familiar to them. During testing, in addition to these 12 familiar stimuli, a set of 12 novel stimuli (six monkey and six flower) was also presented. The set of novel stimuli changed every day. The categorization task performed by the monkey involved a stimulus presentation generated at will by the monkey's action of depressing both levers while fixating for $500 \mathrm{~ms}$ at a fixation spot at the center of a computer screen. Stimuli were presented randomly from the set of 12 flower and 12 monkey stimuli at one of the seven coherence levels for $200 \mathrm{~ms}$. Following image presentation, the monkey had a period of $1200 \mathrm{~ms}$ to categorize the image by releasing one of the levers, left for monkey, or right for flower. Once trained, the monkeys could complete their responses within $600 \mathrm{~ms}$ even under scopolamine. Less than $0.5 \%$ of their responses had latencies greater than $600 \mathrm{~ms}$. Thus the $1200 \mathrm{~ms}$ response window was not restricting their performance.

There were 11 experiments with scopolamine (seven with D07 and four with K03) and nine experiments with butyl-scopolamine (three with D07 and six with K03). Since experiments with only saline were carried out on alternate days, there were 40 test days in total. Minimum intervals were 2 days between scopolamine injections and 3 days between butyl-scopolamine injections. Butylscopolamine experiments began about 3 weeks after the end of the scopolamine experiments to avoid releasing through competitive antagonism scopolamine still bound in the organism. There was a single injection per day and testing began $30 \mathrm{~min}$ after injection. Monkeys performed at least 1100 complete trials per day, that is trials when the monkeys responded within $1200 \mathrm{~ms}$ of the onset of the response cue (range: 1138-2478 trials). Approximately half of the trials were with novel and half with familiar stimuli, half and half in each category, apportioned into the seven coherence levels. The presentation of the stimuli was randomized with respect to familiarity, category, and coherence, only constrained by a condition

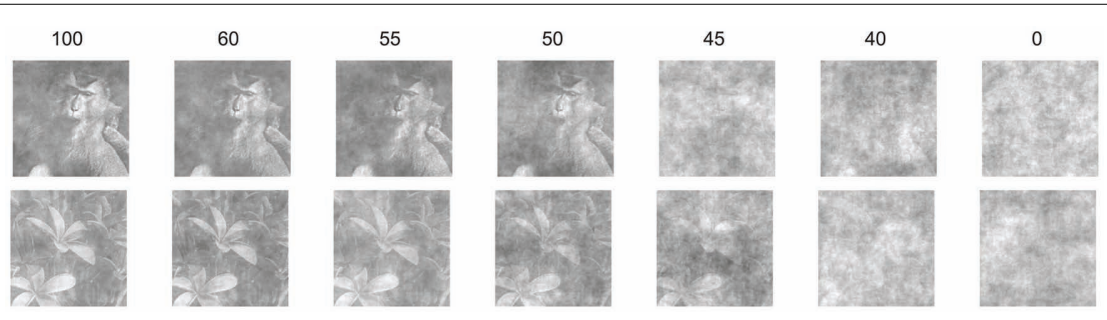

FIGURE 2 | Parametric interpolation with random phases of one monkey and one flower exemplar. Numbers on top of each image refer to \% coherence. At 0 level, the image therefore contains only noise. 


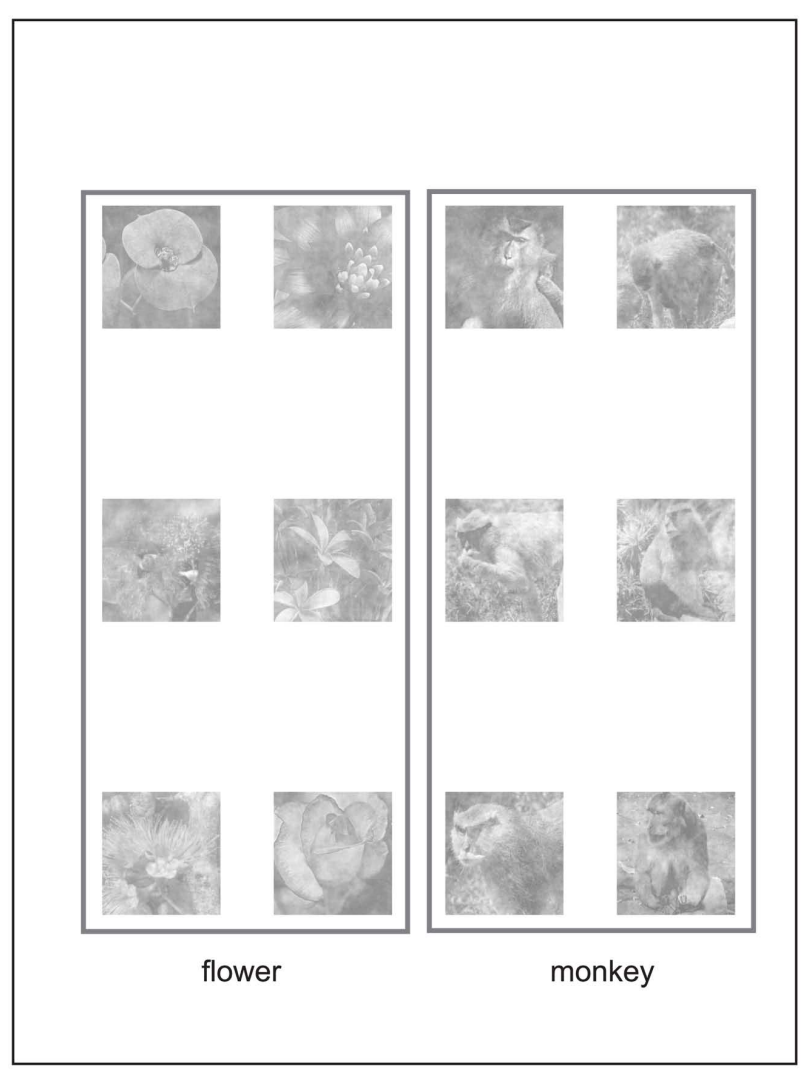

FIGURE 3 | Set of 12 familiar stimuli ( $100 \%$ coherence).

that by 1680 trials responses were required from the monkey for 840 stimuli in each category and each familiarity level, with 240 stimuli in each coherence level. So by 1680 trials, 60 responses were required for every combination of category, familiarity, and coherence level.

\section{STATISTICAL ANALYSIS OF BEHAVIORAL DATA}

For each experiment, psychometric functions were computed in which the proportion of correct responses was plotted against the noise level for each of the four conditions: familiar or unfamiliar stimuli with or without scopolamine using the psignifit routine (Wichmann and Hill, 2001a,b). The same procedure was used to fit behavioral performance under butyl-scopolamine. Confidence limits were computed and a univariate ANOVA was performed (factors: subject, treatment, familiarity, coherence). ANOVAs were carried out both for the probability of a correct response and for latency of response. Response latencies were calculated from the time the stimulus was turned off and the response cue came on, when the macaques could respond by releasing one of the levers.

In some experiments one of the monkeys used a strategy of releasing the levers at random or mostly at random, which would still allow him to obtain reward $50 \%$ of the time. To avoid potential confounds, if performance was impaired during an experiment to less than $60 \%$ average correct for the highest coherence levels, the experiments (three experiments) were not used for further analysis, other than for the response latency measurements.

\section{RESULTS}

The results are presented in three subsections. The effects of scopolamine on categorization performance with either saline or butyl-scopolamine as a control are presented in the first subsection. To assess the effects of scopolamine on categorization performance, psychometric functions were calculated, and we compared the effects of the drug/control on the proportion of correct responses using analysis of variance and associated statistical analyses. The main finding was that categorization of novel stimuli was impaired in both subjects whereas scopolamine had no effect on the categorization of familiar stimuli. In the second subsection, categorization response latencies are compared with or without scopolamine. The effects of scopolamine on other observed parameters, such as pupil dilation and juice consumption, are presented in the third subsection.

\section{CATEGORIZATION PERFORMANCE}

In both monkeys, scopolamine impaired the categorization of novel stimuli, when categorization performance was compared to performance following injection of saline (Figure 4). A four factor ANOVA was carried out (Table 1). Factors were 1. Stimulus coherence (six levels), 2. Drug treatment (scopolamine, butyl-scopolamine or saline), 3. Familiarity (novel or familiar), 4. Subject: D07 or K03.

There was a very highly significant ANOVA $[F(95,408)=40.34$, $\left.p=3.689 \mathrm{e}^{-157}\right]$. Additionally, there was a very highly significant interaction of drug treatment $\times$ familiarity which indicates that at least one of the drugs affected performance for at least one of the familiarity levels (indeed for the novel stimuli, as will be seen further down). The treatment $\times$ familiarity $\times$ coherence interaction was not significant $[F(15,408)=66.74, p=0.072]$. It can be concluded that not only familiarity and drug treatment but also their interaction were independent of coherence. Therefore, as indicated also by the presence of a downward rather than a rightward shift of the psychometric curve, perceptual sensitivity remained similar but categorization performance changed.

Data satisfied the normality requirements for ANOVA (using Kolmogorov-Smirnov and Shapiro-Wilk tests) but overall did not satisfy equality of variances [Levene's test, $F(95,408)=2.291$, $\left.p=1.12 \mathrm{e}^{-8}\right]$. For that reason, three post hoc tests were selected to assess the results of the ANOVA that do not assume equality of variances: the Games-Howell, Dunnet T3, and Tamhane post hoc tests. All three post hoc tests gave the same level of significance for all comparisons.

Paired $t$-tests also confirmed that scopolamine and butylscopolamine affected categorization differently for familiar vs. novel stimuli (Table 2). Specifically, scopolamine but not butylscopolamine impaired the categorization of novel stimuli, whereas in the case of the categorization of familiar stimuli, the effects of scopolamine and butyl-scopolamine were not statistically different according to the post hoc testing (although paired $t$-tests suggested a difference). Post hoc testing also pointed out that the highest three stimulus coherence levels $(55,60$, and $100 \%$ coherence) were homogeneous and did not differ statistically from each other.

How much greater was the impairment under scopolamine to that caused by butyl-scopolamine alone (due to its peripheral actions)? Since the highest three coherence levels were statistically 
homogeneous, we used these three levels to quantify the relative effect size of scopolamine on categorization performance in the two monkeys, by calculating the difference in performance between scopolamine vs. saline and butyl scopolamine vs. saline for the three highest coherence levels (Table 1). We carried out a new ANOVA with the difference between the two drugs as the dependent variable (Table 2 ). The results indicated that the

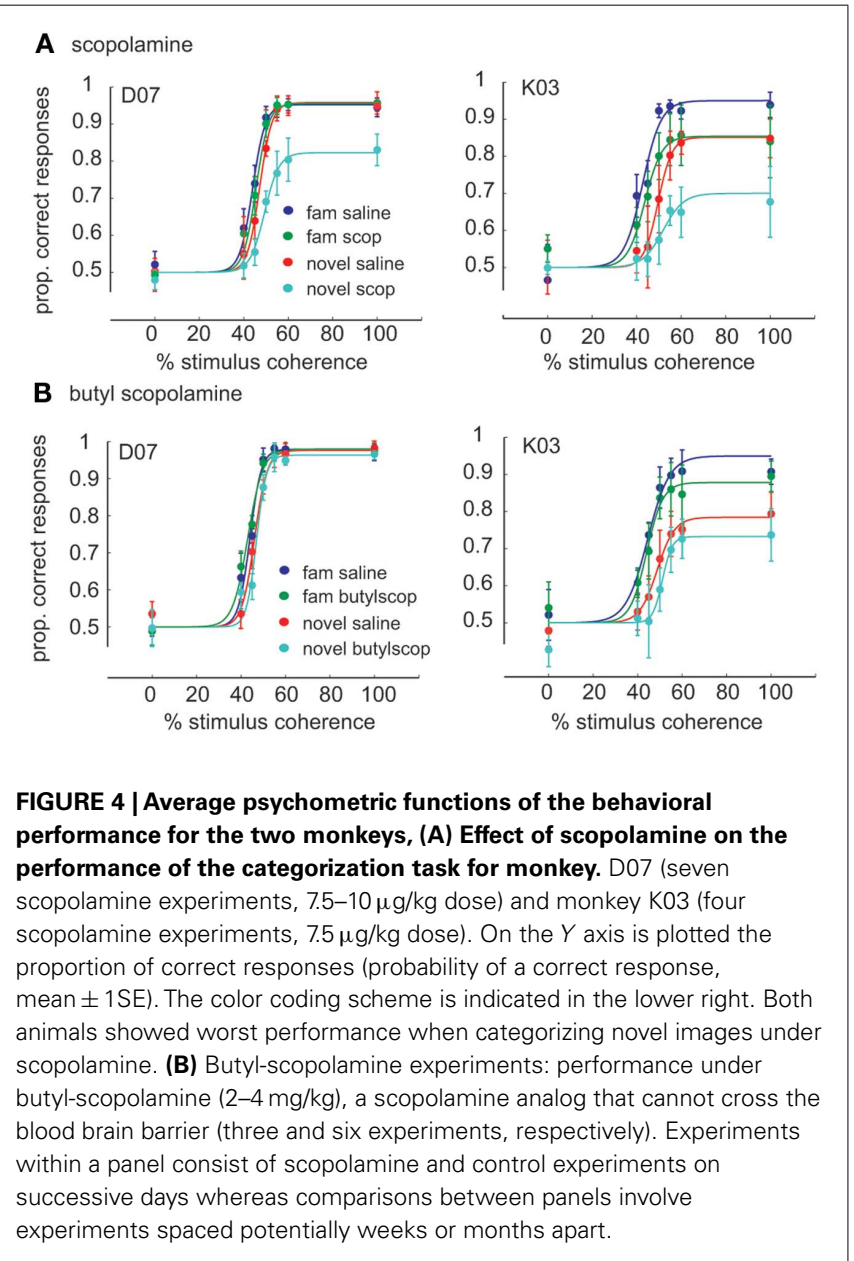

factor familiarity had a very highly significant effect [ANOVA, $\left.F(1,118)=31.07, p=1.60 \mathrm{e}^{-7}\right]$ as did the factor "treatment," i.e., whether the monkeys received scopolamine or butyl-scopolamine $\left[F(1,118)=60.25, p=3.35 \mathrm{e}^{-12}\right]$. This effect did not depend on the subject (monkey) as there were no significant interactions between subject $x$ familiarity or subject $x$ treatment. This analysis also confirms the conclusions of our earlier findings from the ANOVA with all six coherence levels. A summary of these results is presented in Figure 5. The comparisons of drug vs. saline are plotted in Figure 5A and the comparisons of scopolamine vs. butyl-scopolamine in Figure 5B. Compared to butyl-scopolamine, scopolamine led to an overall $11.4 \%$ impairment for monkey K03 and an overall $15.4 \%$ impairment for monkey D07 in the categorization of novel stimuli (with 50\% impairment in correct performance as the maximum). It can be concluded that both monkeys were most impaired, in relation to pharmacological controls, when they had to categorize novel stimuli under scopolamine (Figures 4,5).

\section{RESPONSE LATENCY}

In addition to the effect of scopolamine on the proportion of correct trials, scopolamine affected the latency of the animals' responses in the task (Figure 6). Without scopolamine, average response latency for the $100 \%$ coherence stimuli was $309 \pm 9 \mathrm{~ms}$ (mean $\pm \mathrm{SE}$ ) for monkey D07 and $304 \pm 12 \mathrm{~ms}$ (mean \pm SE) for monkey K03. The latency of response to the stimuli, when a correct response was made, was inversely correlated with coherence and was longer for novel stimuli, presumably a case of speed-accuracy trade-off. Scopolamine increased response latency compared to saline in both monkeys, D07 [ANOVA, $F(1,140)=8.922, p=0.00333$ ] and K03 [ANOVA, $\left.F(1,140)=16.483, p=8.132 \mathrm{e}^{-5}\right]$. Scopolamine's effect in increasing response latency was not specific for novel stimuli and could reflect its role in cue detection and executive processes.

Indeed, no factor interactions were significant; despite an overall trend, one could not argue that scopolamine delayed specifically the categorization of novel stimuli. In monkey D07, however, when only the three most coherent levels were used for the ANOVA, the scopolamine $\times$ familiarity interaction was highly significant. The latency for the categorization of novel stimuli under scopolamine

Table 1 | Paired $t$-tests of categorization performance of drug treatment (scopolamine or butyl-scopolamine) vs. saline (paired experiments were conducted on alternate days).

Paired differences

df

D07 scop nov - D07 sal nov

D07 scop fam - D07 sal fam

D07 butylscop nov - D07 sal nov

D07 butylscop fam - D07 sal fam

K03 scop nov - K03 sal nov

K03 scop fam - K03 sal fam

K03 butylscop nov - K03 sal nov

K03 butylscop fam - K03 sal fam
SE

Mean SE

$\begin{array}{cl}-10.8306122 & 1.157665 \\ -1.04897959 & 0.601105 \\ -0.39285714 & 1.653239 \\ 0.471428571 & 0.893374 \\ 9.903571429 & 2.031423 \\ -5.9 & 1.890123 \\ -4.08571429 & 1.125208 \\ -1.72857143 & 1.317316\end{array}$

48
48
27
27
27
27
41
41

Sig. (two-tailed)

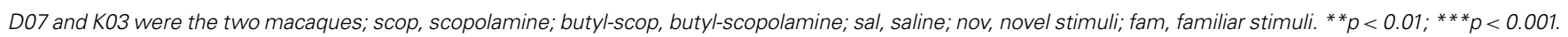


Table 2 |Three-factor univariate ANOVA of correct categorization performance dependent variable: difference in performance (drug-saline) treatment factor: two levels: scopolamine or butyl-scopolamine.

\begin{tabular}{llll}
\hline Source & df & $\boldsymbol{F}$ & Sig. \\
\hline Corrected model & 7 & 21.34863 & $2.26 \mathrm{E}-18^{* * *}$ \\
Intercept & 1 & 134.7247 & $3.04 \mathrm{E}-21^{* * *}$ \\
Subject & 1 & 15.51269 & $0.000139^{* * *}$ \\
Familiarity & 1 & 31.07237 & $1.6 \mathrm{E}-07^{* * *}$ \\
Treatment & 1 & 60.24616 & $3.35 \mathrm{E}-12^{* * *}$ \\
Subject $\times$ familiarity & 1 & 3.043932 & 0.083643 \\
Subject $\times$ treatment & 1 & 1.859905 & 0.175232 \\
Familiarity $\times$ treatment & 1 & 29.66078 & $2.85 \mathrm{E}-07^{* * *}$ \\
Subject $\times$ familiarity $\times$ treatment & 1 & 1.670446 & 0.198724 \\
Error & 118 & & \\
Total & 126 & & \\
\hline
\end{tabular}

${ }^{* * *} p<0.001$

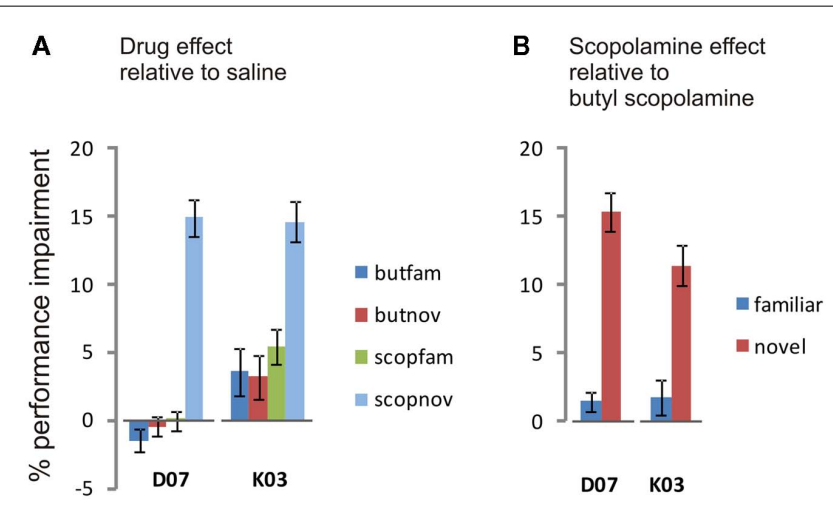

FIGURE 5 | Mean percent impairment of performance for each monkey for the categorization of familiar or novel stimuli compared to control. The means of the three highest coherence levels have been used in making these comparisons. The colors of the bars match the colors of the curves in Figure 4, other than that the control in this case is not saline but butyl-scopolamine. (A) Mean percent performance change (Mean \pm SE) of drug treatment relative to saline. Positive values are for impairment, negative values for improvement. The categorization of novel stimuli was significantly impaired by scopolamine compared to the other treatments which were not significantly different from each other in either monkey. (B) Effect on performance due to the central action of scopolamine: mean percent impairment due to scopolamine after subtracting the mean impairment due to butyl-scopolamine, the peripherally acting analog. On the basis of the result shown in (A), the impairment in the categorization of the familiar stimuli is not significant in either monkey. The categorization of novel stimuli but not of familiar stimuli was significantly impaired by the central actions of scopolamine, based on the butyl-scopolamine control. Statistics in the text.

was longer for coherence level 100 (ANOVA $p=0.009$, paired $t$ test $p=0.005$ ), also for coherence level 60 (ANOVA $p=0.009$, paired $t$-test $p=0.006)$ and it was significant for coherence level 55 (ANOVA $p=0.009$, paired $t$-test $p=0.013$ ). Response latency was shortest when familiar stimuli had to be categorized after injection of saline (Figure 6).

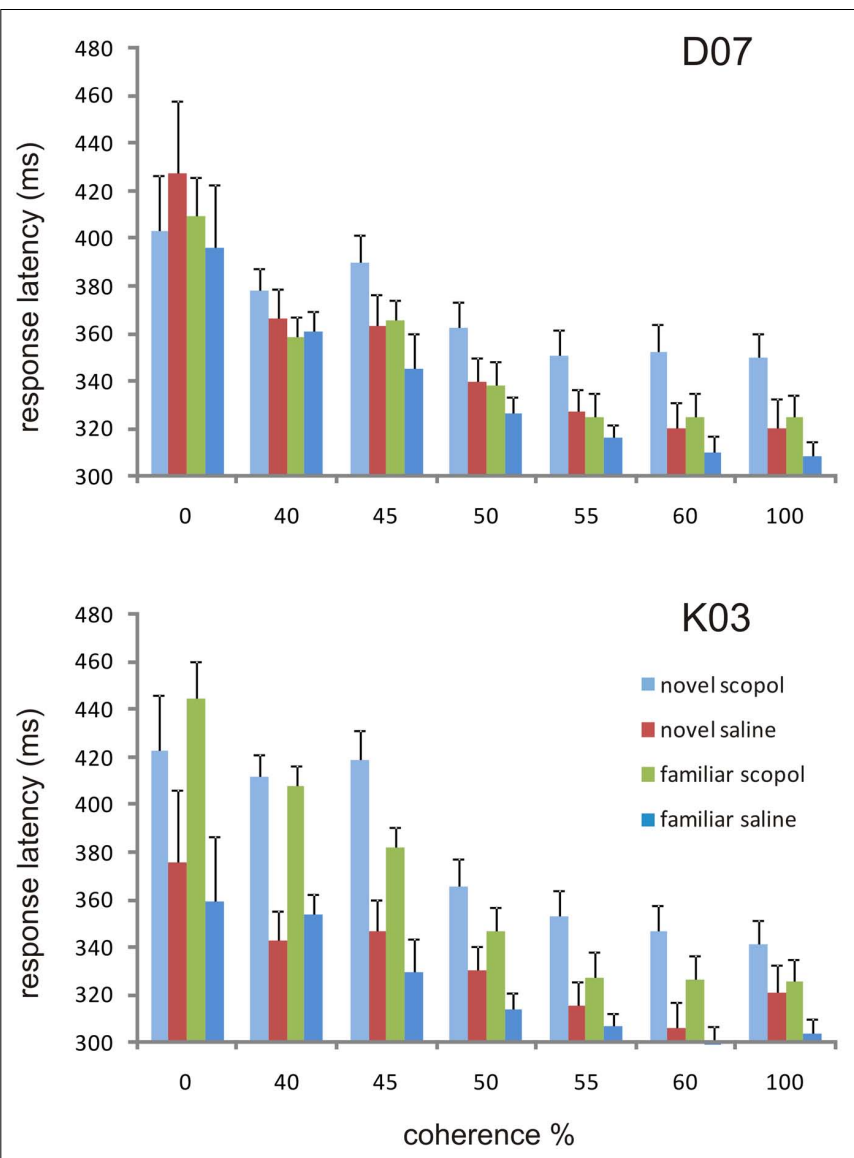

FIGURE 6 | Response latencies under the four different conditions at the seven different coherence levels. Response is measured from the time the stimulus is presented to the time the appropriate lever is released and is shown here for the correct responses only. In monkey D07 (scopolamine drug injections 7.5 and $10 \mu \mathrm{g} / \mathrm{kg}$ combined), response latencies were shorter for the more coherent stimulus levels (ANOVA, $\left.p<1.96 \mathrm{e}^{-14}\right)$. Scopolamine significantly slowed categorization responses for novel stimuli at the more coherent levels (55-100) as compared to categorizing the novel stimuli after injection of saline, while the categorization of familiar stimuli under saline was generally the fastest. In monkey K03 response latencies were also shorter for the more coherent stimulus levels (ANOVA, $p<6.81 \mathrm{e}^{-15}$ ) at the $7.5 \mu \mathrm{g} / \mathrm{kg}$ dose level.

\section{SCOPOLAMINE EFFECTS ON OTHER OBSERVED PARAMETERS}

In addition to its effects on the categorization task, ACh has a variety of other effects. We measured several other parameters that in D07 included pupil diameter, total number of valid trials, percentage of aborted trials, and total volume of juice consumed. Pupil diameter was significantly increased by scopolamine (paired $t$-test $p=0.00012, n=7$ ), total number of valid trials was reduced (paired $t$-test $p=0.0008$, $n=7$ ), percentage of aborted trials following successful fixation was increased (paired $t$-test $p=0.0074, n=7$ ), response latency to the non-degraded stimuli was increased (paired $t$ test $p=0.0013, n=7$ ), experiment duration (hours the monkey worked per day) was increased (paired $t$-test $p=0.00013, n=7$ ), and volume of juice consumed was reduced ( $t$-test $p=0.016$, $n=7)$. 
Some of these parameters were similarly affected by the peripheral agonist butyl-scopolamine: pupil diameter was significantly increased (paired $t$-test $p=0.0040, n=4$ ), total number of valid trials was reduced (paired $t$-test $p=0.0038, n=4$ ). Percentage of aborted trials following successful fixation was unchanged (paired $t$-test $p=0.73, n=4$ ), response latency was unchanged (paired $t$-test $p=0.66, n=4$ ), experiment duration was affected but not significantly (paired $t$-test $p=0.25, n=4$ ), and volume of juice consumed was reduced but not significantly ( $t$-test $p=0.38$, $n=4)$. These effects were, therefore, similar with both drugs. They can best be explained therefore as the result of peripheral parasympathetic depression. Nevertheless, after subtracting the impairment in performance caused by butyl-scopolamine from that caused by scopolamine (e.g., Figure 5) it can be concluded that the central action of scopolamine on the categorization of novel stimuli was independent of peripheral side effects such as pupillary dilation.

\section{DISCUSSION}

We have examined the effects of scopolamine, an antagonist of muscarinic ACh receptors, on visual categorization in macaques. In both animals scopolamine impaired performance in the categorization of novel stimuli, while impairment on the categorization of familiar stimuli was non-significant in both animals, when the peripheral actions of this drug were controlled for. This finding suggests a special role of muscarinic cholinergic mechanisms in the categorization of novel visual information.

Our finding complements previous studies finding a role for acetylcholine in the processing of novel information. Ridley et al. (1984) found no effect of scopolamine on visual discriminations of very familiar objects. Aigner et al. (1987) have proposed that recognition memory (familiarity) is affected by lesions in the NBM, while Wilson and Rolls (1990a) found neurons in the primate substantia inominata (including the NBM) with differential responses between novel and familiar stimuli. Other studies have implicated the basal forebrain cholinergic region in reward associations (Rolls et al., 1980, 1986; Wilson and Rolls, 1990b). Consequently, Masuda et al. (1997) have argued in favor of cholinergic mechanisms of object-reward associations with novel or familiar stimuli in the cholinergic groups of the basal forebrain whose function would have been impaired by scopolamine in our study.

It must be noted that the task the monkeys performed was under executive cognitive control, such that the stimuli appeared on command, following a voluntary depression of the two levers. The monkey did not have to wait for a cue. The cognitive task required only attention to the stimulus itself, while the stimulus was generated at will. Therefore any effects of scopolamine on cue detection and cue-based attention as reported in the literature (see Hasselmo and Sarter, 2011) were bypassed. In this situation, the lack of impairments on the categorization of familiar stimuli suggests that non-cue-driven attention was not affected by scopolamine.

Similar conclusions can be drawn when considering the central effect of scopolamine in impairing performance only for novel stimuli. The implication is that scopolamine did not generally affect attentional mechanisms, perception, decision-making, or basic neuronal mechanisms in ways detrimental to performing this task. The action of scopolamine decreased percent correct performance for novel stimuli beyond any effects caused by the peripherally acting analog butyl-scopolamine, which caused as in the case of a study by Ruotsalainen et al. (2000) an increase in omissions (aborted trials), decrease in the total number of trials completed, and an increased response latency. Additionally, scopolamine affected the categorization of novel stimuli across all coherence levels, indicating a specific learning impairment possibly through an impairment in the acquisition of new information and the storage of new memories. The scopolamine-induced impairment appears to be associated with the categorization of novel stimuli, rather than being a non-specific effect attributable to difficult perceptual judgments, as it was present at all coherence levels.

The specific effect of scopolamine only on the processing of novel stimuli indicates that the effect of scopolamine on object recognition observed in previous studies (Tang et al., 1997; Myers et al., 2002) may be due to an effect on memory encoding rather than due to effects on other cognitive functions such as perception, memory retrieval, decision, or reporting (since the categorization of familiar stimuli was unaffected). This conclusion is in agreement with the conclusions of the Wisconsin General Test Apparatus of Ridley et al. (1984) and the DNMS study by Aigner et al. (1991), that scopolamine has an effect on learning by impairing the encoding of new information in long-term memory. Our findings are also in agreement with the conclusion of Dotigny et al. (2008) that scopolamine does not affect visual acuity in rats, leading the authors to conclude that their study indicates that the basal forebrain cholinergic system is involved in cognitive enhancement or attention during visual learning. Our findings also agree with the conclusion of Miller and Desimone (1993) that scopolamine had no effect on the sensory information conveyed by IT neurons.

There are a number of possibilities based on the literature about the exact involvement of the cholinergic system. One possibility is that scopolamine impairs synaptic plasticity mechanisms facilitated by acetylcholine. For example, activation of muscarinic receptors is required for the induction of corticostriatal long-term potentiation (LTP; Calabresi et al., 1999) and perirhinal long-term depression (LTD; Warburton et al., 2003), actions that can be blocked by scopolamine. Indeed the block of LTD in higher visual area TE of the rat (Warburton et al., 2003) suggests that one role of the cholinergic system may be the acquisition and storage of new information such that novel stimuli may become "familiar." This finding may explain the increased responsiveness under scopolamine of IT neurons to sample stimuli in a DMS task observed by Miller and Desimone (1993). Scopolamine would block the LTD to novel stimuli (only) and therefore neurons in the IT and perirhinal cortex would be expected to have a higher fire rate response to the presentation of novel stimuli (as novel samples in a DMS task) under scopolamine, one of the findings of that study. Additional evidence that $\mathrm{ACh}$ is involved in synaptic plasticity is available from studies in the hippocampus (Drever et al., 2011). Endogenous ACh lowers the threshold for LTP in the hippocampus via a muscarinic receptor dependent mechanism (Ovsepian, 2008). If Hebbian synaptic modification mechanisms are involved, there is additional evidence that would implicate the temporal lobe as the relevant locus. Cholinergic deafferentation of the perirhinal cortex in the rat using saporin immunoglobulin lesions (McGaughy et al., 
2005) and physical lesions to the input from the basal forebrain to area TE and the perirhinal cortex in the macaque (Browning et al., 2010) strongly suggest that object recognition, working memory, and ultimately learning of stimulus reward associations involving novel but not familiar objects depend on cholinergic mechanisms. However, other loci may also be affected, especially as the categorization task involved complex operant conditioning and there is evidence that corticostriatal LTP, an area involved in operant conditioning, depends on local striatal cholinergic activity (Calabresi et al., 1999).

A second possibility is that scopolamine impairs input and gain control in the brain and more so for novel stimuli. A gain-changing action has been hypothesized both for cortical sensory areas as well as for the hippocampus (Sarter et al., 2005; Giocomo and Hasselmo, 2007). This argument is similar to an old hypothesis of a setting of an alert brain state by cholinergic drive (Grossman et al., 1965). A dual mode of action of ACh indeed would involve a combination of both of the above mechanisms (Ovsepian, 2008), with modulation of synaptic plasticity to determine the relative dominance of neuronal inputs while a generalized facilitation of transmission might affect the overall gain for transmission through cortical regions.

A third possibility, especially in relation to the role of the $\mathrm{NBM}$, is that scopolamine affects the categorization especially of novel stimuli via a block of a prefrontal drive (Rasmusson et al., 2007). Cholinergic enhancement produced by the cholinesterase inhibitor physostigmine eliminates the modulation of neural activity by task-difficulty in the prefrontal cortex during working memory (Furey et al., 2008). In such a case, there would be a loss of task-difficulty dependent prefrontal drive. The NBM receives cortical input only from the prefrontal and anterior temporal cortex (Mesulam and Mufson, 1984; Grove, 1988; Gaykema et al., 1991) and is a likely intermediary in the allocation of cortical resources to a difficult task. Therefore scopolamine would presumably block the output of prefrontal drive that could allocate selected cortical/attentional resources to a difficult task, such as the detection of the novel and often noisy stimuli in the categorization task in the present experiments. The exact site of the effect of scopolamine on the categorization task has not yet been established but may indeed depend on forebrain mechanisms with a prefrontal involvement.

It is clear from the fact that the monkeys could categorize novel images with relative accuracy and well above chance, especially in the absence of scopolamine, that the monkeys had learned correctly to form the categories for which they had been trained. The experiment as designed cannot address satisfactorily the question whether the effect of scopolamine was on learning about new objects or on generalizing into better, more inclusive categories. That some object-based learning of the images was involved is expected by the indistinctness of the low-coherence stimuli. At least when their coherence was around the psychophysical threshold, information encoding, and short-term memory would be required (Lewandowsky, 2011) and these are processes needed for new object learning. Categorization, nevertheless, involves generalizing over representations of several objects. The question can be raised whether it was object learning or a generalization to novel exemplars that was affected by scopolamine during this task.
Within this context, there are two hypotheses about how categorization operates, either via new exemplar learning or via the application of categorization rules independent of new learning. According to the first view, categorization involves category generalization analogous to the process of generalizing to several views of the same object, similar to the mechanism of how object learning occurs (Logothetis and Pauls, 1995; Booth and Rolls, 1998). The underlying associational mechanisms for object view generalization (object learning) and category object generalization (category learning) may indeed be quite similar (Soto and Wasserman, 2010). There is evidence that categorization learning uses image fragments qualitatively similar to those used in object learning (Hegdé et al., 2008; Kromrey et al., 2010) and that information for both object identity and object category is available in the same brain region, the IT cortex (see Sigala and Logothetis, 2002; Buckley and Sigala, 2010). Nonetheless, our experiments were not designed to discriminate between these two possible mechanisms for categorization.

Notwithstanding the question of whether object or category learning was impaired by scopolamine at the low-coherence levels, categorization performance for novel stimuli remained impaired even at higher coherence levels. The downward shift in the psychophysical curve for the categorization of novel stimuli by scopolamine tends to favor the interpretation that the antimuscarinic action of scopolamine caused an impairment in categorization at all levels. This would favor a role of acetylcholine in cortical plasticity at levels (and cortical sites) located higher in the visual hierarchy. Nevertheless, here the role of ACh could be analogous to its role in plasticity of the tuning properties of primary sensory neurons (e.g., Bakin and Weinberger, 1996; Kilgard and Merzenich, 1998; Warburton et al., 2003; Weinberger, 2003; Froemke et al., 2007; Kang and Vaucher, 2009).

It must be concluded that already formed object and category representations were unaffected by the antimuscarinic action of scopolamine but that the monkeys were less able to assign novel stimuli as further exemplars into pre-existing categories. It cannot be ascertained on the basis of these results whether the stability of categorization rules or internal "concepts" (Bruner et al., 1967 ) is completely unaffected by muscarinic actions. However, it is likely that already established object category rules and exemplars remained stable, as the categorization of familiar images was not impaired by scopolamine.

\section{CONCLUSION}

In conclusion, scopolamine through its central antagonism of muscarinic ACh receptors affected primarily the categorization of novel stimuli. The process of visual categorization, and in particular the assignment of novel exemplars to a given category, can be disrupted via an inhibition of central muscarinic receptors.

\section{ACKNOWLEDGMENTS}

This research was supported by a German Federal Ministry for Education and Research (Bundesministerium für Bildung und Forschung - BMBF) grant to Dr. Gregor Rainer (Grant number BMBF 01EV0701) and a Ph. D. fellowship from the SFB 550 of the University of Tuebingen for Stefanie Liebe as well as the Max Planck Society. 


\section{REFERENCES}

Aigner, T. G., and Mishkin, M. (1986). The effects of physostigmine and scopolamine on recognition memory in monkeys. Behav. Neural Biol. $45,81-87$.

Aigner, T. G., Mitchell, S. J., Aggleton, J. P., DeLong, M. R, Struble, R. G., Price, D. L., Wenk, G. L., and Mishkin, M. (1987). Effects of scopolamine and physostigmine on recognition memory in monkeys with ibotenic-acid lesions of the nucleus basalis of Meynert. Psychopharmacology (Berl.) 9, 292-300.

Aigner, T. G., Walker, D. L., and Mishkin, M. (1991). Comparison of the effects of scopolamine administered before and after acquisition in a test of visual recognition memory in monkeys. Behav. Neural Biol. 55, 61-67. [Erratum in: Behav. Neural Biol. 55, 380].

Arendt, T., Bigl, V., and Arendt, A. (1984). Neurone loss in the nucleus basalis of Meynert in CreutzfeldtJakob Disease. Acta Neuropathol. 65, 85-88.

Arendt, T., Bigl, V., Arendt, A., and Tennstedt, A. (1983). Loss of neurons in the nucleus basalis of Meynert in Alzheimer's disease, paralysis agitans and Korsakoff's disease. Acta Neuropathol. 61, 101-108.

Bakin, J. S., and Weinberger, N. M. (1996). Induction of a physiological memory in the cerebral cortex by stimulation of the nucleus basalis. Proc. Natl. Acad. Sci. U.S.A. 93, 11219-11224.

Booth, M. C., and Rolls, E. T. (1998). View-invariant representations of familiar objects by neurons in the inferior temporal visual cortex. Cereb. Cortex 8, 510-523.

Browning, P. G., Gaffan, D., Croxson, P. L., and Baxter, M. G. (2010). Severe scene learning impairment, but intact recognition memory, after cholinergic depletion of inferotemporal cortex followed by fornix transection. Cereb. Cortex 20, 282-293.

Bruner, J., Goodnow, J. J., and Austin, G. A. (1967). A Study of Thinking. New York: Science Editions.

Buckley, M. J., and Sigala, N. (2010). Is top-down control from prefrontal cortex necessary for visual categorisation? Neuron 66, 471-473.

Calabresi, P., Centonze, D., Gubellini, P., and Bernardi, G. (1999). Activation of M1-muscarinic like receptors is required for the induction of corticostriatal LTP. Neuropharmacology 38, 323-326.

Deco, G., and Thiele, A. (2010). Attention - oscillations and neuropharmacology. Eur. J. Neurosci. 30, 347-354.
Dotigny, F., Ben Amor, A. Y., Burke, M., and Vaucher, E. (2008). Neuromodulatory role of acetylcholine in visually-induced cortical activation: behavioural and neuroanatomical correlates. Neuroscience 154, 1607-1618.

Drever, B. D., Riedel, G., and Platt, B. (2011). The cholinergic system and hippocampal plasticity. Behav. Brain Res. 221, 505-514.

Ebert, U., Oertel, R., Wesnes, K. A., and Kirch, W. (1998). Effects of physostigmine on scopolamineinduced changes in quantitative electroencephalogram and cognitive performance. Hum. Psychopharmacol. 13, 199-210.

Fransen, E, Tahvildari, B., Egorov, A. V., Hasselmo, M. E., and Alonso, A. A. (2006). Mechanism of graded persistent cellular activity of entorhinal cortex layer V neurons. Neuron 49, 735-746.

Fredrickson, A., Snyder, P. J., Cromer, J., Thomas, E., Lewis, M., and Maruff, P. (2008). The use of effect sizes to characterise the nature of cognitive change in psychopharmacological studies: an example with scopolamine. Hum. Psychopharmacol. 23, 425-436.

Froemke, R. C., Merzenich, M. M., and Schreiner, C. E. (2007). A synaptic memory trace for cortical receptive field plasticity. Nature 450, 425-429.

Furey, M. L., Ricciardi, E., Schapiro, M. B., Rapoport, S. I., and Pietrini, P. (2008). Cholinergic enhancement eliminates modulation of neural activity by task difficulty in the prefrontal cortex during working memory. Neuropsychopharmacology 33, 913-923.

Gaykema, R. P., van Weeghel, R., Hersh, L. B., and Luiten, P. G. (1991). Prefrontal cortical projections to the cholinergic neurons in the basal forebrain. J. Comp. Neurol. 303, 563-583.

Giocomo, L., and Hasselmo, M. (2007). Neuromodulation by glutamate and acetylcholine can change circuit dynamics by regulating the relative influence of afferent input and excitatory feedback. Mol. Neurobiol. 36, 184-200.

Goard, M., and Yang Dan, Y. (2009). Basal forebrain activation enhances cortical coding of natural scenes. Nat. Neurosci. 12, 1444-1449.

Grossman, S. P., Peters, R. H., Freedman, P. E., and Willer, H. I. (1965). Behavioural effects of cholinergic stimulation of the thalamic reticular formation. J. Comp. Physiol. Psychol.59, 57-65.

Grove, E. A. (1988). Neural associations of the substantia innominata in the rat: afferent connections. J. Comp. Neurol. 277, 315-346.

Heims, H. C., Critchley, H. D., Martin, N. H., Jäger, H. R., Mathias, C. J., and Cipolotti, L. (2006). Cognitive functioning in orthostatic hypotension due to pure autonomic failure. Clin. Auton. Res. 16, 113-120.

Hasselmo, M. E., and Sarter, M. (2011). Modes and models of forebrain cholinergic neuromodulation of cognition. Neuropsychopharmacology 36, 52-73.

Hegdé, J., Bart, E., and Kersten, D. (2008). Fragment-based learning of visual object categories. Curr. Biol. 18, 597-601.

Herrero, J. L., Roberts, M. J., Delicato, L. S., Gieselmann, M. A., Dayan, P., and Thiele, A. (2008). Acetylcholine contributes through muscarinic receptors to attentional modulation in V1. Nature 454, 1110-1114.

Kang, J. I., and Vaucher, E. (2009). Cholinergic pairing with visual activation results in long-term enhancement of visual evoked potentials. PLoS ONE 4, e5995. doi:10.1371/journal.pone.0005995

Kilgard, M. P., and Merzenich, M. M. (1998). Cortical Map Reorganisation Enabled by Nucleus Basalis Activity. Science 279, 1714-1718.

Kromrey, S., Maestri, M., Hauffen, K. Bart, E., and Hegdé, J. (2010). Fragment-based learning of visual object categories in non-human primates. PLoS ONE 5:e15444. doi:10.1371/journal.pone.0015444

Levy, A. I. (1996). Muscarinic acetylcholine receptor expression in memory circuits: implications for treatment of Alzheimer disease. Proc. Natl. Acad. Sci. U.S.A. 93, 13541-13546.

Lewandowsky, S. (2011). Working memory capacity for categorisation: Individual differences and modeling. J. Exp. Psychol. Learn. Mem. Cogn. 37, 720-738.

Liebe, S., Fischer, E., Logothetis, N. K. and Rainer, G. (2009). Color and shape interactions in the recognition of natural scenes by human and monkey observers. J. Vis. 5, 14.1-16.

Logothetis, N. K., and Pauls, J. (1995). Psychphysical and physiological evidence for viewer-centered object representations in the primate. Cereb. Cortex 5, 270-288.

Masuda, R., Fukuda, M., Ono, T., and Endo, S. (1997). Neuronal responses at the sight of objects in monkey basal forebrain subregions during operant visual tasks. Neurobiol. Learn. Mem. 67, 181-196.

McGaughy, J., Kaiser, T., and Sarter, M. (1996). Behavioural vigilance following infusions of 192 IgG-saporin into the basal forebrain: selectivity of the behavioural impairment and relation to cortical AChE-positive fiber density. Behav. Neurosci. 110, 247-265.

McGaughy, J., Koene, R. A., Eichenbaum, H., and Hasselmo, M. E. (2005). Cholinergic deafferentation of the entorhinal cortex in rats impairs encoding of novel but not familiar stimuli in a delayed nonmatch-to-sample task. J. Neurosci. 25, 10273-10281.

Mesulam, M.-M., and Mufson, E. J. (1984). Neural inputs into the nucleus basalis of the substantia innominata (Ch4) in the rhesus monkey. Brain 107, 253-274.

Miasnikov, A. A., Chen, J. C., Gross, N., Poytress, B. S., and Weinberger, N. M. (2008). Motivationally neutral stimulation of the nucleus basalis induces specific behavioural memory. Neurobiol. Learn. Mem. 90 125-137.

Miasnikov, A. A., Chen, J. C., and Weinberger, N. M. (2009). Behavioural memory induced by stimulation of the nucleus basalis: effects of contingency reversal. Neurobiol. Learn. Mem. 91, 298-309.

Miasnikov, A. A., McLin, D. III., and Weinberger, N. M. (2001) Muscarinic dependence of nucleus basalis induced conditioned receptive field plasticity. Neuroreport 12, 1537-1542.

Miller, E. K., and Desimone, R. (1993). Scopolamine affects shortterm memory but not inferior temporal neurons. Neuroreport 4 81-84.

Myers, T. M., Galbicka, G., Sipos, M. L., Varadi, S., Oubre, J. L., and Clark, M. G. (2002). Effects of anticholinergics on serial-probe recognition accuracy of rhesus macaques (Macaca mulatta). Pharmacol. Biochem. Behav. 73, 829-834.

Nielsen, K. J., Logothetis, N. K., and Rainer, G. (2006). Discrimination strategies of humans and rhesus monkeys for complex visual displays. Curr. Biol. 16, 814-820.

Ovsepian, S. V. (2008). Differential cholinergic modulation of synaptic encoding and gain control mechanisms in rat hippocampus. Neurosci. Res. 61, 92-98.

Parikh, V., and Sarter, M. (2008). Cholinergic mediation of attention: contributions of phasic and tonic increases in prefrontal cholinergic activity. Ann. N. Y. Acad. Sci. 1129, 225-235.

Pepeu, G., and Giovannini, M. G. (2004). Changes in acetylcholine extracellular levels during cognitive processes. Learn. Mem. 11, 21-27. 
Perry, E. K. (1988). Alzheimer's disease and acetylcholine. Br. J. Psychiatry 152, 737-740.

Plakke, B., Ng, C. W., and Poremba, A. (2008). Scopolamine impairs auditory delayed matching-to-sample performance in monkeys. Neurosci. Lett. 438, 126-130.

Posner, M. I. (1980). Orienting of attention. Q. J. Exp. Psychol. 32, 3-25.

Posner, M. I., Snyder, C. R. R., and Davidson, B. J. (1980). Attention and the detection of signals. J. Exp. Psychol. 109, 160-174.

Rainer, G., Lee, H., and Logothetis, N. K. (2004). The effect of learning on the function of monkey extrastriate visual cortex. PLoS Biol. 2, 44. doi:10.1371/journal.pbio.0020044

Rasmusson, D. D., Smith, S. A., and Semba, K. (2007). Inactivation of prefrontal cortex abolishes cortical acetylcholine release evoked by sensory or sensory pathway stimulation in the rat. Neuroscience 149, 232-241.

Richardson, R. T., and DeLong, M. R. (1988). A reappraisal of the functions of the nucleus basalis of Meynert. Trends Neurosci. 11, 264-267.

Ridley, R. M., Bowes, P. M., Baker, H. F., and Crow, T. J. (1984). An involvement of acetylcholine in object discrimination learning and memory in the marmoset. Neuropsychologia 22, 253-263.

Roberts, A. C., Robbins, T. W., Everitt, B. J., Jones, G. H., Sirkia, T. E., Wilkinson, J., and Page, K. (1990). The effects of excitotoxic lesions of the basal forebrain on the acquisition, retention and serial reversal of visual discriminations in marmosets. $\mathrm{Neu}$ roscience 34, 311-329.

Rolls, E. T., Burton, M. J., and Mora, F. (1980). Neurophysiological analysis of brain-stimulation reward in the monkey. Brain Res. 194, 339-357.

Rolls, E. T., Murzi, E., Yaxley, S., Thorpe, S. J., and Simpson, S. J. (1986). Sensory-specific satiety: food-specific reduction in responsiveness of ventral forebrain neurons after feeding in the monkey. Brain Res. 368, 79-86.

Rosier, A. M., Cornette, L., Dupont, P., Bormans, G., Mortelmans, L., and Orban, G. A. (1999). Regional brain activity during shape recognition impaired by a scopolamine challenge to encoding. Eur. J. Neurosci. 11, 3701-3714.

Ruotsalainen, S., Miettinen, R., MacDonald, E., Koivisto, E., and Sirviö, J. (2000). Blockade of muscarinic, rather than nicotinic, receptors impairs attention, but does not interact with serotonin depletion. Psychopharmacology (Berl.) 148, 111-123.

Sarter, M., and Bruno, J. P. (2000). Cortical cholinergic inputs mediating arousal, attentional processing and dreaming: differential afferent regulation of the basal forebrain by telencephalic and brainstem afferents. Neuroscience 95, 933-952.

Sarter, M., Bruno, J. P., and Givens, B. (2003). Attentional functions of cortical cholinergic inputs: what does it mean for learning and memory? Neurobiol. Learn. Mem. 80, 245-256.

Sarter, M., Hasselmo, M. E., Bruno, J. P., and Givens, B. (2005). Unraveling the attentional functions of cortical cholinergic inputs: interactions between signal-driven and cognitive modulation of signal detection. Brain Res. Brain Res. Rev. 48, 98-111.

Sarter, M., and Parikh, V. (2005). Choline transporters, cholinergic transmission and cognition. Nat Rev. Neurosci. 6, 48-56.

Savage, L. M., Pitkin, S. R., and Knitowski, K. M. (1999). Rats exposed to acute pyrithiamine-induced thiamine deficiency are more sensitive to the amnestic effects of scopolamine and MK-801: examination of working memory, response selection, and reinforcement contingencies. Behav. Brain Res. 104, 13-26.

Sigala, N., and Logothetis, N. K. (2002). Visual categorisation shapes feature selectivity in the primate temporal cortex. Nature 415, 318-320.

Sillito, A. M., and Kemp, J. A. (1983) Cholinergic modulation of the functional organisation of the cat visual cortex. Brain Res. 289, 143-155.

Soto, F. A., and Wasserman, E. A. (2010) Error-driven learning in visual categorisation and object recognition: a common-elements model. Psychol. Rev. 117, 349-381.

Taffe, M. A., Weed, M. R., and Gold, L. H. (1999). Scopolamine alters rhesus monkey performance on a novel neuropsychological test battery. Cogn. Brain Res. 8, 203-212.

Tang, Y., Mishkin, M., and Aigner, T. G. (1997). Effects of muscarinic blockade in perirhinal cortex during visual recognition. Proc. Natl. Acad. Sci. U.S.A. 94, 12667-12669.

Thomas, E., Snyder, P. J., Pietrzak, R. H., Jackson, C. E., Bednar, M. and Maruff, P. (2008). Specific impairments in visuospatial working and short-term memory following low-dose scopolamine challenge in healthy older adults. Neuropsychologia 46, 2476-2484.

Voytko, M. L., Olton, D. S., Richardson, R. T., Gorman, L. K., Tobin, J. R., and Price, D. L. (1994). Basal forebrain lesions in monkeys disrupt attention but not learning and memory. J. Neurosci. 4, 167-186.

Warburton, E. C., Koder, T., Cho, K. Massey, P. V., Duguid, G., Baker, G. R. I., Aggleton, J. P., Bashir, Z. I., and Brown, M. W. (2003). Cholinergic neurotransmission is essential for perirhinal cortical plasticity and recognition memory. Neuron 38, 987-996.

Weinberger, N. M. (2003). The nucleus basalis and memory codes: auditory cortical plasticity and the induction of specific, associative behavioural memory. Neurobiol. Learn. Mem. 80, 268-284.

Wichmann, F. A., and Hill, N. J. (2001a) The psychometric function: I. Fitting, sampling and goodness-of-fit. Percept. Psychophys. 63, 1293-1313.
Wichmann, F. A., and Hill, N. J. (2001b). The psychometric function: II. Bootstrap-based confidence intervals and sampling. Percept. Psychophys. 63, 1314-1329.

Wilson, F. A., and Rolls, E. T. (1990a). Neuronal responses related to the novelty and familiarity of visual stimuli in the substantia innominata, diagonal band of Broca and periventricular region of the primate basal forebrain. Exp. Brain Res. 80, 104-120.

Wilson, F. A. W., and Rolls, E. T. (1990b). Neuronal responses related to reinforcement in the primate basal forebrain. Brain Res. 509, 213-231.

Zinke, W., Roberts, M. J., Guo, K., McDonald, J. S., Robertson, R., and Thiele, A. (2006). Cholinergic modulation of response properties and orientation tuning of neurons in primary visual cortex of anaesthetised Marmoset monkeys. Eur. J. Neurosci. 24, 314-328.

Conflict of Interest Statement: The authors declare that the research was conducted in the absence of any commercial or financial relationships that could be construed as a potential conflict of interest.

Received: 26 August 2011; accepted: 12 October 2011; published online: 15 November 2011.

Citation: Aggelopoulos NC, Liebe S, Logothetis NK and Rainer G (2011) Cholinergic control of visual categorization in macaques. Front. Behav. Neurosci. 5:73. doi: 10.3389/fnbeh.2011.00073 Copyright (c) 2011 Aggelopoulos, Liebe, Logothetis and Rainer. This is an openaccess article subject to a non-exclusive license between the authors and Frontiers Media SA, which permits use, distribution and reproduction in other forums, provided the original authors and source are credited and other Frontiers conditions are complied with. 